\title{
Changes in prognostic factors for patients with hepatocellular carcinoma underwent transarterial chemoembolization with the transition of the time: Child-Pugh class, Albumin-Bilirubin grade, and then
}

\author{
Takumi Kawaguchi ${ }^{1}$, Shigeo Shimose ${ }^{1}$, Sakura Yamamura ${ }^{1}$, Dan Nakano ${ }^{1}$, Masatoshi Tanaka ${ }^{2}$, \\ Takuji Torimura ${ }^{1}$
}

${ }^{1}$ Division of Gastroenterology, Department of Medicine, Kurume University School of Medicine, Kurume, Japan; ${ }^{2}$ Department of Gastroenterology and Hepatology, Yokokura Hospital, Miyama, Japan

Correspondence to: Takumi Kawaguchi, MD, PhD. Division of Gastroenterology, Department of Medicine, Kurume University School of Medicine, Kurume, Japan. Email: takumi@med.kurume-u.ac.jp.

Provenance and Peer Review: This article was commissioned by the editorial office, Annals of Translational Medicine. The article did not undergo external peer review.

Comment on: Zhao S, Zhang T, Li H, et al. Comparison of albumin-bilirubin grade versus Child-Pugh score in predicting the outcome of transarterial chemoembolization for hepatocellular carcinoma using time-dependent ROC. Ann Transl Med 2020;8:538.

Submitted May 01, 2020. Accepted for publication May 18, 2020.

doi: 10.21037/atm-2020-112

View this article at: http://dx.doi.org/10.21037/atm-2020-112

Prognostic factor is crucial for the management of hepatocellular carcinoma (HCC) patients. Based on large international databases, the albumin-bilirubin (ALBI) grade has originally been developed and is independently associated with overall survival in HCC patients (1). The usefulness of ALBI grade in predicting overall survival has been validated in patients with HCC underwent liver resection (2), radiofrequency ablation (3), and tyrosine kinase inhibitors $(4,5)$. The prognostic impact of ALBI grade has also been reported in patients with intermediatestage HCC underwent transarterial chemoembolization (TACE) (6). In addition, Zhao et al. recently demonstrated that ALBI grade was better than Child-Pugh class in terms of stratifying prognosis in patients with HCC underwent TACE (7). We summarize the recent progress in prediction of overall survival for patients with HCC underwent TACE by focusing on ALBI grade. We also discuss the future perspective of systematic predictive factors for patients with HCC underwent TACE.

Liver function is associated with overall survival of patients with HCC (8). To assess liver function, Child-Pugh class has commonly been used and is better predictor of overall survival than model for end-stage liver disease score in patients with HCC underwent TACE (8). However, Child-Pugh class seems to have several limitations as followings. First, Child-Pugh class includes subjective parameters such as ascites and hepatic encephalopathy. Second, ascites and hepatic encephalopathy can be modified by medications. Third, prothrombin activity, a constitutive parameter of Child-Pugh class, can be affected by anticoagulant therapy. Fourth, each variable is assigned the same weight for the assessment of liver function. It is also important to re-recognize that Child-Pugh class is not applicable for patients with chronic hepatitis, because Child-Pugh class is designed for patients with liver cirrhosis. Recent progress in treatment for chronic hepatitis $\mathrm{C}$ and $\mathrm{B}$ virus infections causes an increase in the prevalence of noncirrhotic patients with HCC. Moreover, the proportion of patients with non-alcoholic fatty liver disease-related HCC has been increasing and their liver function is often preserved (9). Thus, the shortcomings of Child-Pugh class and recent changes in characteristics of patients with HCC lead a question regarding an accuracy of Child-Pugh class as a prognostic factor for patients with HCC.

In order to assess liver function for patients with HCC, Johnson et al. developed ALBI score, which is based on 
objective parameters such as serum levels of albumin and total bilirubin (1). The formula is $\{\log 10$ [total bilirubin level $(\mathrm{mg} / \mathrm{dL}) \times 17.1] \times 0.66\}+[$ serum albumin level $(\mathrm{g} / \mathrm{dL}) \times 10 \times$ $-0.085]$ and the ALBI score is classified into three grades as followings: grade $1(\leq-2.6)$, grade $2(>-2.6$ to $\leq-1.39)$, and grade 3 (>-1.39) (1). Johnson et al. also reported that Child-Pugh class A can be divided into ALBI grade 1 and 2 (1), and there is a 10 -month difference in overall survival between the ALBI grade 1 and 2 (1). Thus, ALBI grade is thought to accurately predict liver function as well as overall survival in patients with HCC. In fact, the majority of patients with HCC underwent TACE are classified as Child-Pugh class A (10). Peng et al. performed a systematic review to compare the prognostic ability of ALBI score versus Child-Pugh class in patients with HCC (11). They found that ALBI grade showed better than Child-Pugh class for predicting the prognosis in patients with HCC (11). However, it remains unclear if ALBI grade has higher predictive ability than Child-Pugh class in predicting overall survival of patients with HCC underwent TACE. Recently, Zhao et al. has compared the performance of ALBI grade and Child-Pugh class for predicting overall survival in HCC patients who underwent TACE (7). As a result, they enrolled 221 cirrhotic patients with Child-Pugh class A and HCC underwent TACE. In this study, time-dependent receiver operating characteristic curves have been employed to assess the predictive power of diagnostic markers for time-dependent disease outcomes (7). They clearly showed that the discriminatory ability of ALBI grade was better than Child-Pugh class for overall survival (7).

The recent study by Zhao et al. demonstrated the superiority of ALBI grade than Child-Pugh class for predicting overall survival of patients with HCC underwent TACE (7). However, this study also has several limitations. First, this was a retrospective single center study, suggesting a selection bias. Secondary, the most of subjects were patients with hepatitis B viral-related HCC. It remains unclear if the superiority of ALBI grade is seen in patients with the non-viral hepatitis-related HCC including nonalcoholic fatty liver disease-related HCC, which is becoming a major etiology of HCC $(9,12,13)$. Moreover, in this study, ALBI score $>-2.6$ to $\leq-1.39$ was classified to the ALBI grade 2, which covers a wide range of liver impairment. While, Hiraoka et al. proposed the modified ALBI (mALBI) grade, which divides ALBI grade 2 into two subgrades (grade $2 \mathrm{a} ;>-2.6$ to $\leq-2.27$ and grade $2 \mathrm{~b}$; $>-2.27$ to $\leq-1.39$ ) (5). Hiraoka et al. conducted a multicenter analysis, in which 6,649 naïve HCC patients were enrolled and demonstrated that mALBI grade has a better assessment ability for predicting overall survival than ALBI grade and Child-Pugh class (14). In addition, serum levels of albumin and bilirubin are affected by various factors other than liver function. Therefore, more precise model to evaluate liver function are still unmet needs. The platelet-albumin-bilirubin (PALBI) grade has recently been developed to reflect the effect of portal hypertension based on the ALBI grade (15). Although ALBI grade differentiates Child-Pugh class A patients into two groups, PALBI grade differentiates Child-Pugh class A patients into three groups (16). Jaruvongvanich et al. investigate the prognostic impact of 6 non-invasive models including, Child-Pugh class, ALBI, and PALBI in patients with HCC. They found that the PALBI is the most accurate prognostic model among 6 models to predict HCC recurrence and mortality (17). In addition, Lee et al. evaluated the ability of PALBI and ALBI grades to predict overall survival according to treatment modality in patients with HCC (18). They found that the ALBI grade showed high predictive ability for overall survival than PALBI in patients underwent surgical resection or radiofrequency ablation (18). While, the PALBI grade showed high predictive ability for overall survival than ALBI in patients underwent TACE or supportive care (18). Thus, proper use of the mALBI and PALBI grades based on the treatment modality may enable accurate determination of the prognosis of patients with HCC.

Recent advances in prevention, diagnosis, and treatment for HCC lead a long-term survival of patients with HCC (19). As a result, the prevalence of aged patients with HCC has been increasing (20). Aging is known to alter immunity, and Li et al. performed a meta-analysis with 5,280 HCC patients from 22 studies and investigated an impact of neutrophil-to-lymphocyte ratio (NLR), which is an index of systemic inflammation and immune suppression on prognosis of patients with HCC (21). They demonstrated that NLR was identified as an independent factor associated with the presence of tumor vascular invasion and poor prognosis in patients with HCC underwent TACE (21). In addition, the prevalence of malnutrition increases according to aging. The controlling nutritional status (CONUT) score is a simple objective assessment tool of nutritional status (22). The CONUT score is based on following three variables: serum albumin level, total cholesterol level, and total lymphocyte count (22). The CONUT score has recently been reported as an independent prognostic factor for patients with HCC treated with lenvatinib in a 


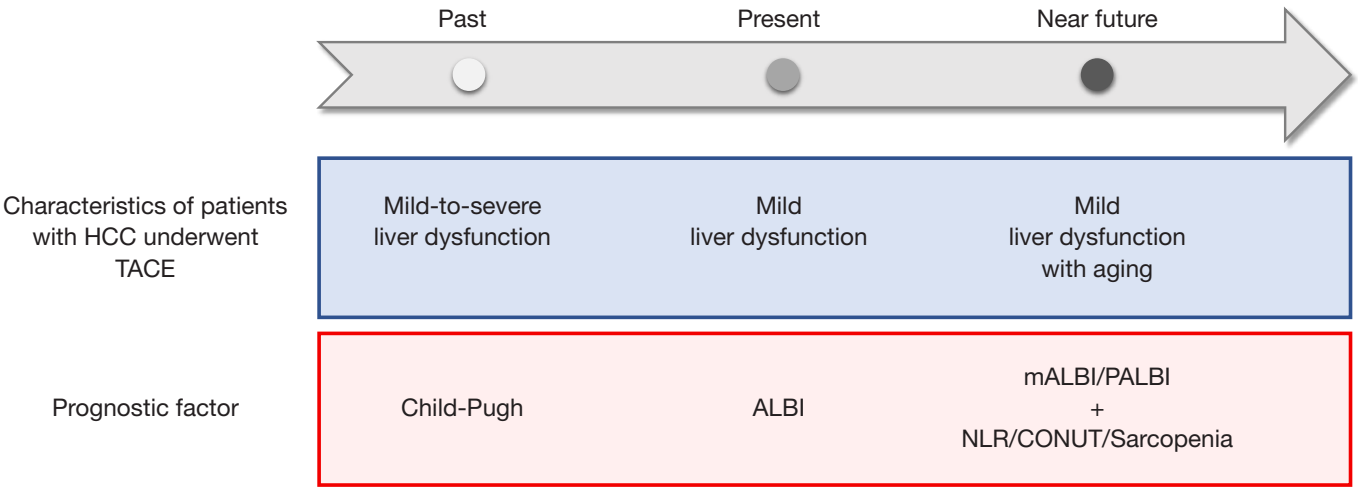

Figure 1 Changing in characteristics of patients with HCC underwent TACE and prognostic factor across the ages. HCC, hepatocellular carcinoma; TACE, transcatheter arterial chemoembolization; ALBI, albumin-bilirubin grade; mALBI, modified ALBI; PALBI, plateletalbumin-bilirubin grade; NLR, neutrophil-to-lymphocyte ratio; CONUT, controlling nutritional status.

multicenter cohort study (22). Moreover, the prevalence of sarcopenia is high in the elderly and sarcopenia is now recognized as an important prognostic factor independent from liver function in patients with HCC (23). Skeletal muscles play crucial roles in glucose, protein, and ammonia metabolisms (23). Moreover, skeletal muscle is known to release cytokines called myokines, which suppress proliferation of cancer cells $(20,23)$. In fact, sarcopenia is a negative prognostic factor in patients with HCC underwent TACE (24). Loss of skeletal muscle mass during TACE is associated with poor prognosis in patients with HCC (25). While, in-hospital exercise maintains skeletal muscle mass during TACE $(26,27)$ and has been reported to improve prognosis in patients with HCC underwent TACE (23). Thus, prognostic factors have been changing with the transition of the time.

In conclusion, ALBI grade is more suitable for assessment of liver function than Child-Pugh class in patients with HCC underwent TACE. Because the most of such patients show mild liver dysfunction classified as Child-Pugh class A at present (Figure 1). In addition, to improve the predictive accuracy, modification of ALBI such as mALBI and PALBI is better to be employed. Moreover, to adapt to the high prevalence of aging in patients with HCC, there is no doubt that assessment for general condition including immunity, nutrition, and skeletal muscle mass will become more important. Thus, NLR, CONUT, and sarcopenia should be considered as prognostic factors in near future (Figure 1).

\section{Acknowledgments}

Funding: This editorial was supported by AMED under
Grant Number JP20fk0210040.

\section{Footnote}

Conflicts of Interest: All authors have completed the ICMJE uniform disclosure form (available at http://dx.doi. org/10.21037/atm-2020-112). Dr. TK reports personal fees from MSD. K.K., personal fees from Mitsubishi Tanabe Pharma Corporation, personal fees from Otsuka Pharmaceutical Co., Ltd., outside the submitted work. The other authors have no conflicts of interests to declare.

Ethical Statement: The authors are accountable for all aspects of the work in ensuring that questions related to the accuracy or integrity of any part of the work are appropriately investigated and resolved.

Open Access Statement: This is an Open Access article distributed in accordance with the Creative Commons Attribution-NonCommercial-NoDerivs 4.0 International License (CC BY-NC-ND 4.0), which permits the noncommercial replication and distribution of the article with the strict proviso that no changes or edits are made and the original work is properly cited (including links to both the formal publication through the relevant DOI and the license). See: https://creativecommons.org/licenses/by-nc-nd/4.0/.

\section{References}

1. Johnson PJ, Berhane S, Kagebayashi C, et al. Assessment of liver function in patients with hepatocellular carcinoma: a new evidence-based approach-the ALBI grade. J Clin 
Oncol 2015;33:550-8.

2. Wang YY, Zhong JH, Su ZY, et al. Albumin-bilirubin versus Child-Pugh score as a predictor of outcome after liver resection for hepatocellular carcinoma. Br J Surg 2016;103:725-34.

3. Hiraoka A, Kumada T, Hirooka M, et al. A better method for assessment of hepatic function in hepatocellular carcinoma patients treated with radiofrequency ablation: Usefulness of albumin-bilirubin grade. Hepatol Res 2018;48:E61-7.

4. Tada T, Kumada T, Toyoda H, et al. Impact of albuminbilirubin grade on survival in patients with hepatocellular carcinoma who received sorafenib: An analysis using time-dependent receiver operating characteristic. J Gastroenterol Hepatol 2019;34:1066-73.

5. Hiraoka A, Michitaka K, Kumada T, et al. Validation and Potential of Albumin-Bilirubin Grade and Prognostication in a Nationwide Survey of 46,681 Hepatocellular Carcinoma Patients in Japan: The Need for a More Detailed Evaluation of Hepatic Function. Liver Cancer 2017;6:325-36.

6. Lanza E, Muglia R, Bolengo I, et al. Survival analysis of 230 patients with unresectable hepatocellular carcinoma treated with bland transarterial embolization. PLoS One 2020;15:e0227711.

7. Zhao S, Zhang T, Li H, et al. Comparison of albuminbilirubin grade versus Child-Pugh score in predicting the outcome of transarterial chemoembolization for hepatocellular carcinoma using time-dependent ROC. Ann Transl Med 2020;8:538.

8. Brown DB, Fundakowski CE, Lisker-Melman M, et al. Comparison of MELD and Child-Pugh scores to predict survival after chemoembolization for hepatocellular carcinoma. J Vasc Interv Radiol 2004;15:1209-18.

9. Kawaguchi T, Tokushige K, Hyogo H, et al. A Data Mining-based Prognostic Algorithm for NAFLD-related Hepatoma Patients: A Nationwide Study by the Japan Study Group of NAFLD. Sci Rep 2018;8:10434.

10. Lee YH, Hsu CY, Chu CW, et al. A new Child-TurcottePugh class 0 for patients with hepatocellular carcinoma: determinants, prognostic impact and ability to improve the current staging systems. PLoS One 2014;9:e99115.

11. Peng Y, Wei Q, He Y, et al. ALBI versus Child-Pugh in predicting outcome of patients with HCC: A systematic review. Expert Rev Gastroenterol Hepatol 2020. doi: 10.1080/17474124.2020.1748010.

12. Kudo M, Izumi N, Kubo S, et al. Report of the 20th Nationwide follow-up survey of primary liver cancer in
Japan. Hepatol Res 2020;50:15-46.

13. Tateishi R, Uchino K, Fujiwara N, et al. A nationwide survey on non-B, non-C hepatocellular carcinoma in Japan: 2011-2015 update. J Gastroenterol 2019;54:367-76.

14. Hiraoka A, Kumada T, Tsuji K, et al. Validation of Modified ALBI Grade for More Detailed Assessment of Hepatic Function in Hepatocellular Carcinoma Patients: A Multicenter Analysis. Liver Cancer 2019;8:121-9.

15. Sonohara F, Yamada S, Tanaka N, et al. Comparison of non-invasive liver reserve and fibrosis models: Implications for surgery and prognosis for hepatocellular carcinoma. Hepatol Res 2019;49:1305-15.

16. Lu LH, Zhang YF, Mu-Yan C, et al. Platelet-albuminbilirubin grade: Risk stratification of liver failure, prognosis after resection for hepatocellular carcinoma. Dig Liver Dis 2019;51:1430-7.

17. Jaruvongvanich V, Sempokuya T, Wong L. Is there an optimal staging system or liver reserve model that can predict outcome in hepatocellular carcinoma? J Gastrointest Oncol 2018;9:750-61.

18. Lee SK, Song MJ, Kim SH, et al. Comparing various scoring system for predicting overall survival according to treatment modalities in hepatocellular carcinoma focused on Platelet-albumin-bilirubin (PALBI) and albuminbilirubin (ALBI) grade: A nationwide cohort study. PLoS One 2019; 14:e0216173.

19. Kulik L, El-Serag HB. Epidemiology and Management of Hepatocellular Carcinoma. Gastroenterology 2019;156:477-91.e1.

20. Kawaguchi T, Yoshio S, Sakamoto Y, et al. Impact of Decorin on the Physical Function and Prognosis of Patients with Hepatocellular Carcinoma. J Clin Med 2020;9:936.

21. Li S, Feng X, Cao G, et al. Prognostic significance of inflammatory indices in hepatocellular carcinoma treated with transarterial chemoembolization: A systematic review and meta-analysis. PLoS One 2020;15:e0230879.

22. Shimose S, Kawaguchi T, Iwamoto H, et al. Controlling Nutritional Status (CONUT) Score is Associated with Overall Survival in Patients with Unresectable Hepatocellular Carcinoma Treated with Lenvatinib: A Multicenter Cohort Study. Nutrients 2020;12:E1076.

23. Hashida R, Kawaguchi T, Koya S, et al. Impact of cancer rehabilitation on the prognosis of patients with hepatocellular carcinoma. Oncol Lett 2020,19:2355-67.

24. Loosen SH, Schulze-Hagen M, Bruners P, et al. Sarcopenia Is a Negative Prognostic Factor in Patients 
Undergoing Transarterial Chemoembolization (TACE) for Hepatic Malignancies. Cancers (Basel) 2019;11:1503.

25. Fujita $M$, Takahashi A, Hayashi $M$, et al. Skeletal muscle volume loss during transarterial chemoembolization predicts poor prognosis in patients with hepatocellular carcinoma. Hepatol Res 2019;49:778-86.

26. Koya S, Kawaguchi T, Hashida R, et al. Effects of in-

Cite this article as: Kawaguchi T, Shimose S, Yamamura S, Nakano D, Tanaka M, Torimura T. Changes in prognostic factors for patients with hepatocellular carcinoma underwent transarterial chemoembolization with the transition of the time: Child-Pugh class, Albumin-Bilirubin grade, and then. Ann Transl Med 2020;8(17):1045. doi: 10.21037/atm-2020-112 hospital exercise on liver function, physical ability, and muscle mass during treatment of hepatoma in patients with chronic liver disease. Hepatol Res 2017;47:E22-34.

27. Koya S, Kawaguchi T, Hashida R, et al. Effects of inhospital exercise on sarcopenia in hepatoma patients who underwent transcatheter arterial chemoembolization. J Gastroenterol Hepatol 2019;34:580-8. 